\title{
Kinetics of phytosterol metabolism in neonates receiving parenteral nutrition
}

\author{
T. Hang Nghiem-Rao', Ilker Tunc'2, Alisha M. Mavis' ${ }^{1}$ Yumei Cao', Elizabeth M. Polzin³ ${ }^{3}$ Mary F. Firary ${ }^{4}$, \\ Xujing Wang' ${ }^{2}$, Pippa M. Simpson' ${ }^{1}$ and Shailendra B. Patel ${ }^{5,6}$
}

BACKGROUND: Phytosterols in soybean oil (SO) lipids likely contribute to parenteral nutrition-associated liver disease (PNALD) in infants. No characterization of phytosterol metabolism has been done in infants receiving $\mathrm{SO}$ lipids.

METHODS: In a prospective cohort study, 45 neonates (36 SO lipid vs. 9 control) underwent serial blood sample measurements of sitosterol, campesterol, and stigmasterol. Mathematical modeling was used to determine pharmacokinetic parameters of phytosterol metabolism and phytosterol exposure.

RESULTS: Compared to controls, sO lipid-exposed infants had significantly higher levels of sitosterol and campesterol $(P<0.01)$. During SO lipid infusion, sitosterol and campesterol reached half of steady-state plasma levels within 1.5 and $0.8 \mathrm{~d}$, respectively. Steady-state level was highest for sitosterol $(1.68 \mathrm{mg} / \mathrm{dl})$, followed by campesterol $(0.98 \mathrm{mg} / \mathrm{dl})$, and lowest for stigmasterol $(0.01 \mathrm{mg} / \mathrm{dl})$. Infants born $<28$ wk gestational age had higher sitosterol steady-state levels $(P=0.03)$ and higher area under the curve for sitosterol $(P=0.03)$ during the first $5 \mathrm{~d}$ of $\mathrm{SO}$ lipid $\left(\mathrm{AUC}_{5}\right.$ ) than infants born $\geq 28 \mathrm{wk}$ gestational age.

CONCLUSION: Phytosterols in SO lipid accumulate rapidly in neonates. Very preterm infants receiving SO lipid have higher sitosterol exposure, and may have poorly developed mechanisms of eliminating phytosterols that may contribute to their vulnerability to PNALD.

$\mathbf{P}$ arenteral nutrition-associated liver disease (PNALD) is a well-known complication of parenteral nutrition (PN) in critically ill neonates. While early institution of PN improves growth and long-term neurodevelopmental outcome $(1,2)$, prolonged exposure can lead to potentially devastating, irreversible liver injury (3). Preterm infants are especially vulnerable and are among those at highest risk for severe cholestasis and death $(4,5)$. This serious complication is strongly associated with the dose and duration of parenteral lipids, and plant sterols (phytosterols) in soybean oil (SO) lipid emulsions may contribute to PNALD (6,7). Phytosterols are naturally occurring steroid alcohols unique to plants and components of cell membranes, similar to cholesterol in mammalian species. In animal and in vitro models, phytosterols induce cholestasis (8), cause direct hepatocyte damage $(9,10)$, and antagonize nuclear receptors critical for hepato-protection against bile acid injury and elimination of phytosterols $(10,11)$.

SO lipid emulsions, rich in phytosterols, are the only lipid emulsions approved by the US Food and Drug Administration (12). However, the content of phytosterols in SO lipids are not regulated or reported in formulations. Intravenous administration of SO lipid bypasses protective mechanisms that prevent against phytosterol accumulation in the body. Under normal conditions, intestinal and hepatic ABCG5/G8 transporters are integral for phytosterol elimination. Intestinal ABCG5/ G8 transporters excrete dietary plant sterols into the intestinal lumen to prevent more than $95 \%$ of ingested phytosterols from entering the circulation, and the small amounts allowed entry are then excreted by hepatic ABCG5/G8 into the biliary tract (13). Direct intravenous entry of phytosterols into the circulation will increase hepatic exposure to these potential toxins and challenge normal mechanisms of excretion. The ability of neonates to manage phytosterols in intravenous SO lipid is unknown.

Despite the frequent use of $\mathrm{PN}$, there are no data regarding the kinetics of plant sterol accumulation in humans receiving phytosterol-enriched lipid emulsions. These data are particularly important in preterm and critically ill neonates who are most susceptible to PNALD. We conducted this prospective cohort study to determine the kinetics of phytosterol accumulation in infants receiving SO lipid (Intralipid, Fresenius Kabi, Uppsala, Sweden). We hypothesized that plasma phytosterol concentrations increase during SO lipid infusion, and that the kinetics of phytosterol metabolism and exposure to phytosterols during SO lipid infusion are influenced by gestational age.

\section{RESULTS}

Study Population and Demographics

Of the 56 eligible infants, 36 neonates received SO lipid and 9 control neonates who did not receive SO lipid were enrolled. 
Eleven screened subjects were excluded from study participation (parenteral refusal, $n=2$; genetic or chromosomal defects, $n=4$; metabolic disorder, $n=3$; received SO lipid for $<5 \mathrm{~d}$, $n=2)$. The median gestational age and birth weight for study participants was $33.1 \mathrm{wk}$ (IQR 29.6-36) and $1.79 \mathrm{~kg}$ (IQR $1.22-2.54)$, respectively. Demographic and clinical characteristics for SO lipid-exposed and control groups are shown in Table 1. Compared to controls, SO lipid-exposed infants were significantly smaller at birth, more frequently admitted with a diagnosis of prematurity, and had longer duration of hospitalization. As expected, the control group had earlier initiation of enteral feeds (day 1 (IQR 1-1) vs. day 6 (IQR 2-14), control vs. SO lipid-exposed respectively, $P<0.01$ ), and reached full enteral feeds sooner (day 5 (IQR 4-6) vs. day 18 (IQR 10-26), control vs. SO lipid-exposed respectively, $P<0.01$ ). Sterol concentrations in SO lipid are shown in Table 2, and SO lipid and phytosterol intake of infants in the SO lipid-exposed group are shown in Table 3.

\section{SO Lipid-Exposed vs. Control}

Higher plasma phytosterol concentrations were seen in infants who received SO lipid compared to control infants who did not receive SO lipid. Compared to the control group, SO lipid-exposed infants had significantly higher maximum levels for sitosterol $(2.78 \mathrm{mg} / \mathrm{dl}$ (IQR $1.94-4.17)$ vs. $0.01 \mathrm{mg} / \mathrm{dl}$ (IQR 0.01-0.01), $P<0.01$ ) and campesterol $(2.42 \mathrm{mg} / \mathrm{dl}$ (IQR $1.34-4.31$ ) vs. 0.34 (IQR $0.26-1.13$ ), $P<0.01$ ). Although maximum stigmasterol trended higher in the SO lipid-exposed group, this did not reach statistical significance $(0.40 \mathrm{mg} / \mathrm{dl}$ (IQR $0.01-0.70)$ vs. $0.01 \mathrm{mg} / \mathrm{dl}$ (IQR $0.01-0.01$ ), $P=0.06$ ). Among the SO lipid-exposed, four infants developed cholestasis. Infants with cholestasis had significantly higher maximum sitosterol $(7.80 \mathrm{mg} / \mathrm{dl}$ (IQR $4.49-12.86)$ vs. $2.55 \mathrm{mg} / \mathrm{dl}$ (IQR $1.91-3.44), P<0.01)$ and stigmasterol levels $(2.13 \mathrm{mg} / \mathrm{dl}$ (IQR $1.25-3.57$ ) vs. $0.34 \mathrm{mg} / \mathrm{dl}$ (IQR $0.01-0.58), P<0.01$ ) compared to those without cholestasis.

\section{Phytosterol Kinetics and Exposure}

In order to assess phytosterol accumulation, phytosterol levels after SO lipid initiation until reaching a steady-state plasma concentration were examined. Overall, infants who received SO lipid showed a progressive increase in sitosterol and campesterol to reach steady median concentrations 3-5 d after initiating SO lipid. In contrast, levels of stigmasterol remained at the lowest level of detection. Accumulation of sitosterol reached a steady-state concentration $\left(C_{s s}\right)=1.68 \mathrm{mg} / \mathrm{dl}$ (IQR 1.08-2.43), with time to reach half of steady-state concentration $\left(T_{1 / 2}\right)=1.5 \mathrm{~d}$ (IQR 1.2-1.6), and time between $1 / 2$ and $3 / 4$ of steady-state concentration $\left(T_{1 / 2-3 / 4}\right)=0.5 \mathrm{~d}$ (IQR 0.1-0.8). Campesterol $C_{s s}=0.98 \mathrm{mg} / \mathrm{dl}$ (IQR 0.55-1.30), $T_{1 / 2}=0.8 \mathrm{~d}$ (IQR 0-1.4), and $T_{1 / 2-3 / 4}=1.1 \mathrm{~d}$ (IQR 0.7-2.1). After filtering was applied with a threshold for $R$ square of 0.1 and radius of the confidence interval for $C_{s s}$ of less than 20, little change was seen in the model fit curves. The observed phytosterol concentration vs. time plots and accumulation model fit curves, with and without filtering, are shown in Figure 1a-c
Table 1. Demographic and clinical characteristics of infants in SO lipid-exposed and control groups

\begin{tabular}{|c|c|c|c|}
\hline & $\begin{array}{c}\text { SO lipid- } \\
\text { exposed }(n=36)\end{array}$ & $\begin{array}{l}\text { Control } \\
(n=9)\end{array}$ & $P$ value \\
\hline $\begin{array}{l}\text { Gestational age } \\
\text { (weeks) }\end{array}$ & $32.4(28.6,36)$ & $34(33,37.9)$ & $0.05^{a}$ \\
\hline Birth weight (kg) & $1.46(1.07,2.32)$ & $2.49(1.79,3.01)$ & $0.02^{*, a}$ \\
\hline \multicolumn{4}{|l|}{ Sex } \\
\hline Male & $17(47)$ & $5(56)$ & $0.72^{\mathrm{b}}$ \\
\hline Race/Ethnicity & & & $0.57^{\mathrm{b}}$ \\
\hline $\begin{array}{l}\text { Non-Hispanic } \\
\text { White }\end{array}$ & $22(61)$ & $5(56)$ & \\
\hline $\begin{array}{l}\text { Non-Hispanic } \\
\text { Black }\end{array}$ & $9(25)$ & $2(22)$ & \\
\hline Hispanic & $2(6)$ & $0(0)$ & \\
\hline Other & $3(8)$ & $2(22)$ & \\
\hline \multicolumn{4}{|l|}{ Diagnosis } \\
\hline Prematurity & $32(89)$ & $5(56)$ & $0.04^{*, b}$ \\
\hline SGA & $9(25)$ & $2(22)$ & $>0.99^{\mathrm{b}}$ \\
\hline RDS & $23(64)$ & $7(78)$ & $0.70^{\mathrm{b}}$ \\
\hline PDA & $5(14)$ & $2(22)$ & $0.61^{\mathrm{b}}$ \\
\hline Sepsis & $2(6)$ & $0(0)$ & $>0.99^{b}$ \\
\hline TEF & $2(6)$ & $0(0)$ & $>0.99^{b}$ \\
\hline Gastroschisis & $10(28)$ & $0(0)$ & $0.17^{b}$ \\
\hline $\begin{array}{l}\text { Intestinal atresia or } \\
\text { stenosis }\end{array}$ & $5(14)$ & $0(0)$ & $0.57^{b}$ \\
\hline NEC & $2(6)$ & $0(0)$ & $>0.99^{b}$ \\
\hline Imperforate anus & $1(3)$ & $0(0)$ & $>0.99^{b}$ \\
\hline Omphalocele & $0(0)$ & $1(11)$ & $0.20^{\mathrm{b}}$ \\
\hline $\begin{array}{l}\text { Spontaneous } \\
\text { perforation }\end{array}$ & $3(8)$ & $0(0)$ & $>0.99^{\mathrm{b}}$ \\
\hline Malrotation & $1(3)$ & $0(0)$ & $>0.99^{\mathrm{b}}$ \\
\hline G-tube placement & $1(3)$ & $0(0)$ & $>0.99^{b}$ \\
\hline Volvulus & $1(3)$ & $0(0)$ & $>0.99^{b}$ \\
\hline Cholestasis & $4(11)$ & $0(0)$ & $0.57^{b}$ \\
\hline Gl surgery (yes) & $19(53)$ & $0(0)$ & $<0.01^{*, t}$ \\
\hline
\end{tabular}

Data are presented as median (interquartile range) or $n(\%)$

Gl, gastrointestinal; G-tube, gastrostomy tube; NEC, necrotizing enterocolitis; PDA, patent ductus arteriosus; RDS, respiratory distress syndrome; SGA, small for gestational age; TEF, tracheoesophageal fistula.

aMann-Whitney (Wilcoxon rank-sum) test. ${ }^{b}$ Fisher's exact test. ${ }^{*} P$ value $<0.05$.

Table 2. Sterol concentrations in SO lipid

\begin{tabular}{lc}
\hline & Concentration \\
\hline Sitosterol $(\mathrm{mg} / \mathrm{dl})$ & $19.72(17.15,22.27)$ \\
Campesterol $(\mathrm{mg} / \mathrm{dl})$ & $8.52(6.58,10.06)$ \\
Stigmasterol $(\mathrm{mg} / \mathrm{dl})$ & $7.72(6.04,8.94)$ \\
Cholesterol $(\mathrm{mg} / \mathrm{dl})$ & $34.82(29.96,39.93)$ \\
Total sterols $(\mathrm{mg} / \mathrm{dl})$ & $72.10(60.55,79.20)$ \\
\hline
\end{tabular}

Measured by gas-chromotography - mass spectrometry in our laboratory. Data are presented as median (interquartile range). 
(phytosterol model fit curves for each individual are shown in Supplementary Figures S1-S3). Phytosterol exposure, as evaluated using average area under the plasma concentration versus time curve over the first $5 \mathrm{~d}$ of $\mathrm{SO}$ lipid $\left(\mathrm{AUC}_{5}\right)$ was highest for sitosterol (median $0.74 \mathrm{mg} / \mathrm{dl} \times$ day (interquartile range (IQR) $0.45-1.28$ )), followed by campesterol (median $0.72 \mathrm{mg} / \mathrm{dl} \times$ day (IQR $0.37-1.07)$ ), and lowest for stigmasterol (median $0.01 \mathrm{mg} / \mathrm{dl} \times$ day $(\mathrm{IQR} 0.01-0.04)$ ).

\section{Prematurity and Phytosterol Kinetics}

Clinical data and phytosterol intakes for very preterm (born $<28 \mathrm{wk}$ gestation) and more mature (born $\geq 28 \mathrm{wk}$ gestation) gestational age group categories are compared in Table 4. A gastrointestinal diagnosis on admission was more common in the more mature group ( 0 vs. $60 \%$, very preterm vs. more mature respectively, $P=0.02$ ). No study subjects were

Table 3. SO lipid and phytosterol intake of infants in the SO lipidexposed group $(n=36)$

\begin{tabular}{ll}
\hline & \multicolumn{1}{c}{ Intake } \\
\hline SO lipid & $1(1,1)$ \\
Day of life at SO lipid initiation & $17(8,26)$ \\
Total number of SO lipid days & \\
Sterol intake during first 5 d of SO lipid & $2.96(2.43,3.26)$ \\
Sitosterol (mg/kg per day) & $1.16(0.93,1.39)$ \\
Campesterol (mg/kg per day) & $1.13(0.91,1.29)$ \\
Stigmasterol (mg/kg per day) & $5.00(4.14,5.55)$ \\
Cholesterol $(\mathrm{mg} / \mathrm{kg}$ per day) & \\
Cumulative sterol intake during entire hospitalization & \\
Sitosterol (mg/kg) & $49.90(25.18,78.21)$ \\
Campesterol $(\mathrm{mg} / \mathrm{kg})$ & $20.34(10.18,33.77)$ \\
Stigmasterol $(\mathrm{mg} / \mathrm{kg})$ & $18.66(9.40,29.84)$ \\
Cholesterol $(\mathrm{mg} / \mathrm{kg})$ & $81.66(41.21,130.25)$ \\
\hline
\end{tabular}

Data are presented as median (interquartile range) or $\mathrm{n}(\%)$. diagnosed with short bowel syndrome. Culture-positive sepsis was seen more frequently in the very preterm group, and all episodes of sepsis were of late-onset. Enteral feeds were initiated significantly earlier in the very preterm group (day 2 (IQR 1-2) vs. day 8 (IQR 2-16), very preterm vs. more mature respectively, $P=0.01$ ); however, the time to reach full enteral feeds was similar between groups (day 16 (IQR 9-30) vs. day 18 (IQR 11-25), very preterm vs. more mature respectively, $P=0.91)$. There were no differences in SO lipid or phytosterol intake between groups.

Very preterm infants had higher sitosterol steady-state concentrations than more mature infants $(2.60$ vs. $1.33 \mathrm{mg} / \mathrm{dl}$, respectively, $P=0.03$ ). In addition, sitosterol $\mathrm{AUC}_{5}$ was significantly higher for the very preterm compared to the more mature group $(P=0.03)$. Phytosterol $\mathrm{AUC}_{5}$ for gestational age categories is shown in Figure 2. The difference in sitosterol AUC $_{5}$ between gestational age groups remained significant after inclusion of race, sex, cumulative sitosterol intake for the first $5 \mathrm{~d}$ of $\mathrm{SO}$ lipid, and number of days receiving enteral feeds during the first $5 \mathrm{~d}$ of SO lipid in the model $(P=0.04)$. Unadjusted and adjusted campesterol $\mathrm{AUC}_{5}$ and stigmasterol AUC $_{5}$ were similar between the two groups $(P$ values $>0.05)$.

Median and maximum plasma phytosterol levels were similar between gestational age groups (Table 5). There was no difference in the incidence of cholestasis ( 17 vs. $10 \%$, very preterm vs. more mature, respectively, $P=0.54$ ) or maximum conjugated bilirubin levels $(0.05 \mathrm{mg} / \mathrm{dl}$ (IQR $0-0.7)$ vs. $0.5 \mathrm{mg} / \mathrm{dl}$ (IQR $0-0.8$ ), very preterm vs. more mature, respectively, $P=0.54$ ) between groups. Serum triglyceride levels were higher in the very preterm group $(P=0.01)$. Details regarding clinical laboratory results are presented in Supplementary Table S1.

Medications received by study participants are shown in Table 6. Compared to more mature infants, very preterm infants had significantly higher diuretic $(P<0.01)$ and surfac$\operatorname{tant}(P=0.03)$ therapy. There was also a trend toward more ibuprofen and systemic steroid therapy in the very preterm group, but this did not reach statistical significance $(P=0.07$ for both).
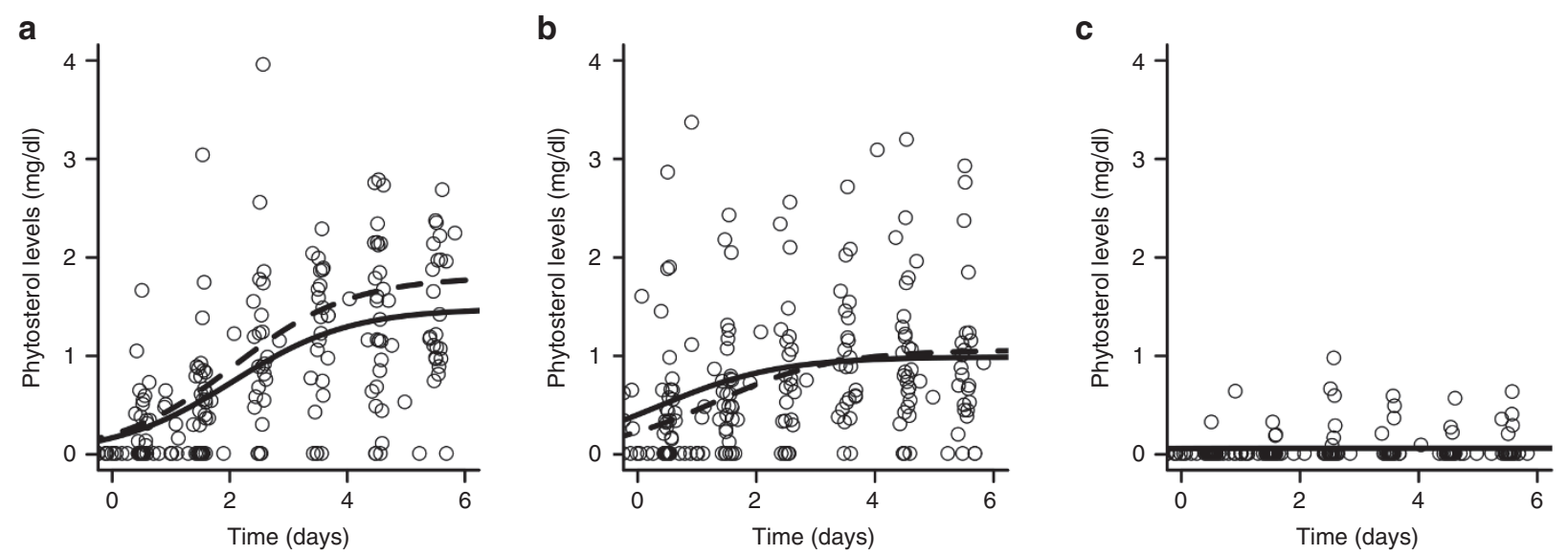

Figure 1. Serial phytosterol levels in infants after the initiation (a-c) of soybean oil lipid. Circles represent individual phytosterol levels. Black lines indicate model fit curve using the median of all individual kinetic parameters for the accumulation of (a) sitosterol, (b) campesterol, and (c) stigmasterol. Dashed lines represent model fit curve using the median of filtered individual fits. 


\section{Articles $\mid$ Nghiem-Rao et al.}

Table 4. Demographic, clinical, and phytosterol intake characteristics of infants in gestational age group categories

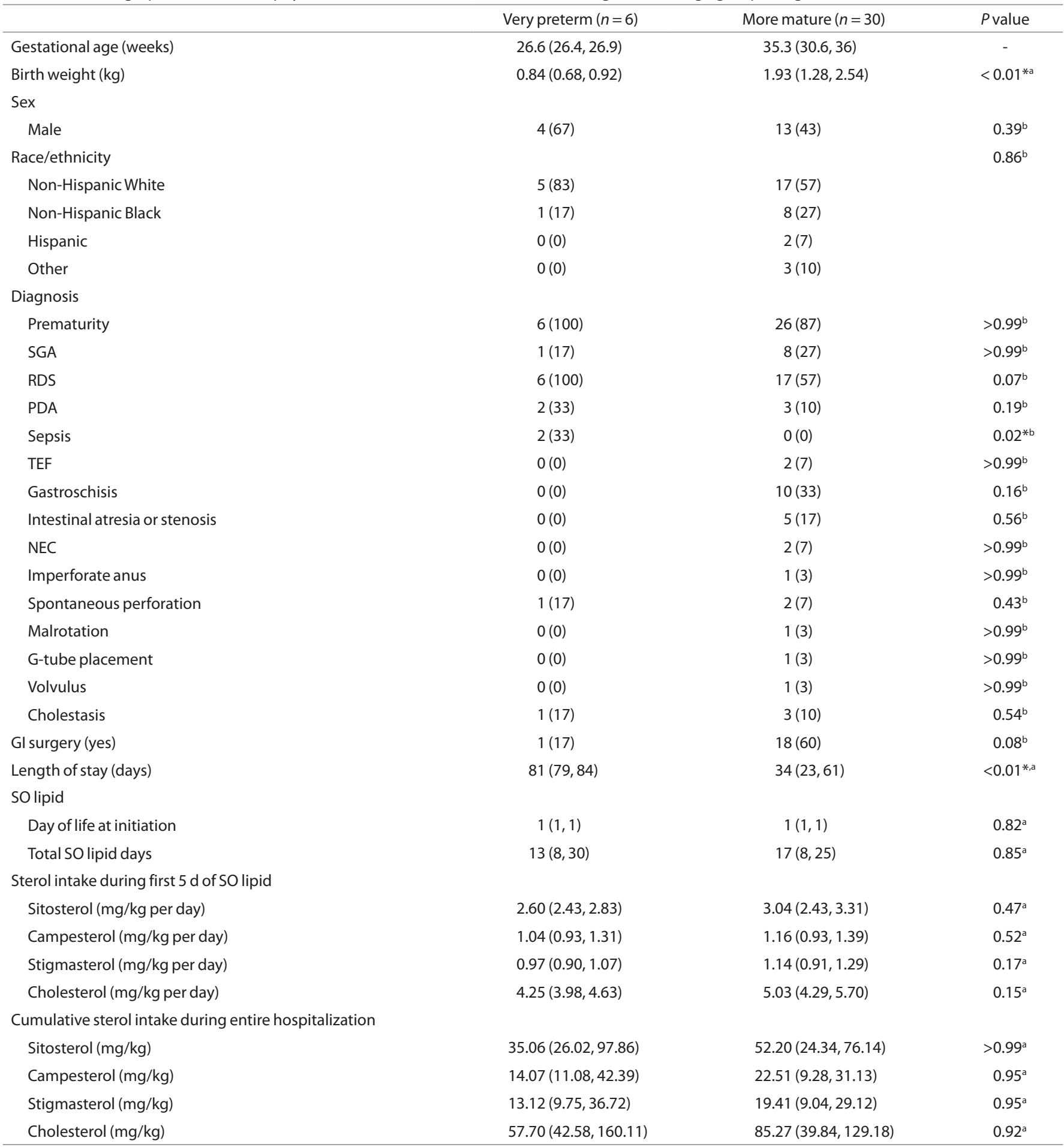

Data are presented as median (interquartile range) or $n(\%)$.

Gl, gastrointestinal; G-tube, gastrostomy tube; NEC, necrotizing enterocolitis; PDA, patent ductus arteriosus; RDS, respiratory distress syndrome; SGA, small for gestational age; TEF, tracheoesophageal fistula.

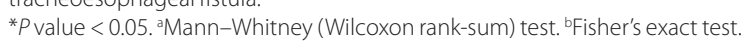

\section{Phytosterols in Patients With Cholestasis}

In all infants with cholestasis, sitosterol, and campesterol levels rose and achieved steady-state prior to an increase in conjugated bilirubin. In contrast, changes in stigmasterol levels were generally preceded by an increase in conjugated bilirubin in all except for one patient. While phytosterol and conjugated bilirubin levels gradually declined after the discontinuation of SO lipid, elevated levels persisted for greater than $2 \mathrm{wk}$. Changes in conjugated bilirubin most closely resembled those of sitosterol. Serial sitosterol, stigmasterol, and conjugated bilirubin levels 
for the four patients with cholestasis are shown in Figure 3. Among the phytosterols, campesterol appeared to exhibit the most variability (data not shown).

\section{DISCUSSION}

This is the first characterization of the kinetics of phytosterol metabolism in humans receiving PN. We demonstrate that neonates receiving SO lipid rapidly accumulate sitosterol and campesterol. Steady-state phytosterol levels are seen within a few days of SO lipid initiation and exceed those of normal individuals who consume dietary phytosterols $(14,15)$. Very preterm infants had higher sitosterol steady-state concentrations and twice the sitosterol exposure as more mature infants in the first $5 \mathrm{~d}$ of SO lipid, suggesting that prematurity affects sterol metabolism.

We found that infants with cholestasis had the highest phytosterol levels. This finding supports the association of elevated phytosterols and PNALD and is consistent with published literature. In clinical studies, elevated phytosterol levels have been correlated with PNALD in infants and children with prolonged PN/SO lipid dependence (6,7). Animal models demonstrated the association between increased phytosterols and elevated serum bile acid levels, lower maximal bile acid accumulation, and significant inhibition of hepatic secretory function (16).

Recent studies have reported the hepatotoxicity of stigmasterol. In hepatic tissue culture, stigmasterol was the most potent farnesoid X receptor (FXR) antagonist among the three predominant phytosterols in soybean oil-based lipid emulsion (10). Using a mouse model, El Kasmi et al. (11) showed that stigmasterol antagonism of the FXR and liver X receptor (LXR) suppressed expression of canalicular bile transporters, contributed to bile acid-induced hepatocyte damage, promoted cholestasis and hepatic macrophage activation, and suppressed hepatic ABCG5/G8 sterol transporters. In infants with PNALD, we found that stigmasterol levels were the lowest among the three phytosterols. Given its biologic potency, it is possible that even low levels of stigmasterol may be sufficient in promoting liver injury. It should be noted, however, that no other phytosterols were tested by El Kasmi et al. and the capacity of other sterols for inducing liver injury is not known. In most cholestatic patients, we found that an increase in stigmasterol above the level of detection was generally observed after a rise in conjugated bilirubin. In contrast, increases in sitosterol and campesterol preceded the rise in conjugated bilirubin. The delayed rise in stigmasterol that we observed suggests that stigmasterol may not be the only phytosterol to cause cholestasis and that exceeding safe thresholds of other phytosterols may contribute to liver injury.

While earlier studies have identified the significance of elevated phytosterols in liver injury, none have previously characterized changes in their concentrations during routine care. Given that all infants receiving soybean oil-based lipid emulsion are exposed to potentially toxic phytosterols, understanding sterol metabolism is important to nutritional management of premature and critically ill infants.

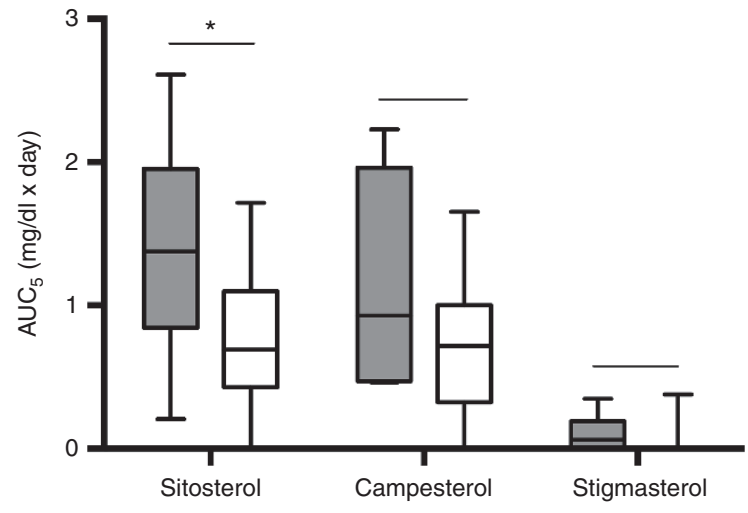

Figure 2. Comparison of unadjusted area under the curve for each phytosterol during the first $5 \mathrm{~d}\left(\mathrm{AUC}_{5}\right)$ of soybean oil lipid between gestational age groups. Gray boxes indicate very preterm group and white boxes indicate more mature group. Box $=25-75 \%$; line inside box = median; error bars $=$ minimum and maximum. ${ }^{*} P=0.03$.

Table 5. Plasma sterol levels of infants in gestational age group categories

\begin{tabular}{lccc}
\hline & Very preterm $(n=6)$ & More mature $(n=30)$ & $P$ value \\
\hline $\begin{array}{l}\text { Sitosterol } \\
\text { (mg/dl) }\end{array}$ & $1.14(0.01,2.08)$ & $1.05(0.31,1.41)$ & $0.92^{\mathrm{a}}$ \\
\multicolumn{1}{c}{ Maximum } & $3.5(2.73,4.37)$ & $2.55(1.91,4.07)$ & $0.46^{\mathrm{a}}$ \\
$\begin{array}{l}\text { Campesterol } \\
\text { (mg/dl) }\end{array}$ & $0.80(0.63,1.13)$ & $0.62(0.38,0.99)$ & $0.36^{\mathrm{a}}$ \\
$\quad$ Maximum & $3.18(2.93,4.23)$ & $2.14(1.27,4.39)$ & $0.23^{\mathrm{a}}$ \\
$\begin{array}{l}\text { Stigmasterol } \\
\text { (mg/dl) }\end{array}$ & $0.01(0.01,0.01)$ & $0.01(0.01,0.01)$ & $0.95^{\mathrm{a}}$ \\
\multicolumn{1}{c}{ Maximum } & $0.41(0.33,1.73)$ & $0.4(0.01,0.69)$ & $0.56^{\mathrm{a}}$ \\
$\begin{array}{l}\text { Cholesterol } \\
\text { (mg/dl) }\end{array}$ & $78.02(57.69,129.49)$ & $71.53(55.2,88.06)$ & $0.46^{\mathrm{a}}$ \\
\multicolumn{1}{c}{ Maximum } & $123.93(104.41,242.38)$ & $101.66(84.22,136.92)$ & $0.09^{\mathrm{a}}$ \\
\hline
\end{tabular}

Data are presented as median (interquartile range).

aMann-Whitney (Wilcoxon rank-sum) test.

Two proteins, ABCG5 and ABCG8, have been identified as key regulators of phytosterols. Both are found in apical membranes of the small intestine and liver where they serve as sterol export pumps to prevent phytosterol accumulation in the body $(17,18)$. In normal individuals who consume phytosterols in their diet, low plasma phytosterol concentrations are primarily due to intestinal ABCG5/G8 that prevent their absorption (13). Administration of intravenous SO lipid bypasses the intestinal barrier mechanism to rely on hepatic ABCG5/G8 for excretion.

Using mathematical modeling to uniquely describe changes in plasma phytosterols in infants exposed to SO lipid, we found that even a short duration of intravenous SO lipid exposure in infants led to accumulation of sitosterol and campesterol to steady-state plasma concentrations that are more than twice those of normal adults who consume phytosterols in their diet $(14,15)$. These findings are consistent with the concept that ABCG5/G8 transporters are significant in preventing dietary absorption of phytosterols. Compared to the other phytosterols, stigmasterol levels remained low. Although the affinity 
Table 6. Medications received by infants in gestational age group categories

\begin{tabular}{lccc}
\hline & $\begin{array}{c}\text { Very preterm } \\
(n=6)\end{array}$ & $\begin{array}{c}\text { More mature } \\
(n=30)\end{array}$ & \begin{tabular}{c}
$P$ value \\
\hline Opiate
\end{tabular} l(17) \\
Muscle relaxant & $2(33)$ & $12(40)$ & $0.39^{\mathrm{a}}$ \\
Ibuprofen & $2(33)$ & $1(3)$ & $>0.99^{\mathrm{a}}$ \\
Acetaminophen & $5(83)$ & $22(73)$ & $0.07^{\mathrm{a}}$ \\
Sedative/hypnotic & $1(17)$ & $11(37)$ & $>0.99^{\mathrm{a}}$ \\
lonotrope & $1(17)$ & $3(10)$ & $0.64^{\mathrm{a}}$ \\
Antihypertensive & $0(0)$ & $3(10)$ & $0.53^{\mathrm{a}}$ \\
Diuretic & $6(100)$ & $5(17)$ & $>0.99^{\mathrm{a}}$ \\
Surfactant & $4(67)$ & $5(17)$ & $<0.01^{*, a}$ \\
PPI & $0(0)$ & $8(27)$ & $0.03^{*, a}$ \\
Antiepileptic & $0(0)$ & $1(3)$ & $0.30^{\mathrm{a}}$ \\
Antibiotic & $6(100)$ & $30(100)$ & $>0.99^{\mathrm{a}}$ \\
Steroid & $2(33)$ & $1(3)$ & $>0.99^{\mathrm{a}}$ \\
Ursodiol & $1(17)$ & $2(7)$ & $0.07^{\mathrm{a}}$ \\
Erythromycin & $1(17)$ & $0(0)$ & $0.43^{\mathrm{a}}$ \\
\hline
\end{tabular}

Data are presented as $n(\%)$.

PPI, proton pump inhibitor.

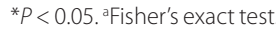

and saturability of ABCG5/G8 by different phytosterols are unknown, it is possible that ABCG5/8 binds stigmasterol more readily for elimination and our findings may reflect a higher stigmasterol clearance relative to other phytosterols.

Despite similar phytosterol intakes in gestational age groups, our data indicate a higher area under the curve for sitosterol, and thus higher exposure to sitosterol, in the youngest infants during the first $5 \mathrm{~d}$ of SO lipid. This may be an important factor in their elevated risk for developing PNALD. The steady-state sitosterol level seen in our very preterm group was similar to those reported in preterm infants $<1,250 \mathrm{~g}$ after $7 \mathrm{~d}$ of a $100 \%$ soybean oil lipid emulsion by Savini et al. (19); however, the steady-state sitosterol level achieved in our more mature group was lower and closer to levels seen in preterm infants $<1,250 \mathrm{~g}$ after $7 \mathrm{~d}$ of alternative lipid emulsions with lower soybean oil lipid content. Even after adjusting for confounders, the area under the curve for sitosterol in the first $5 \mathrm{~d}$ of SO lipid was significantly higher in very preterm infants born $<28 \mathrm{wk}$ compared to more mature infants. A developmental predisposition to altered sterol metabolism is supported by clinical observations of different populations receiving $\mathrm{PN}$ and phytosterolrich diets. Among children receiving prolonged $\mathrm{PN}$ for more than $28 \mathrm{~d}$, higher phytosterol levels were seen in neonates compared to older children (7). Infants given a phytosterol-rich
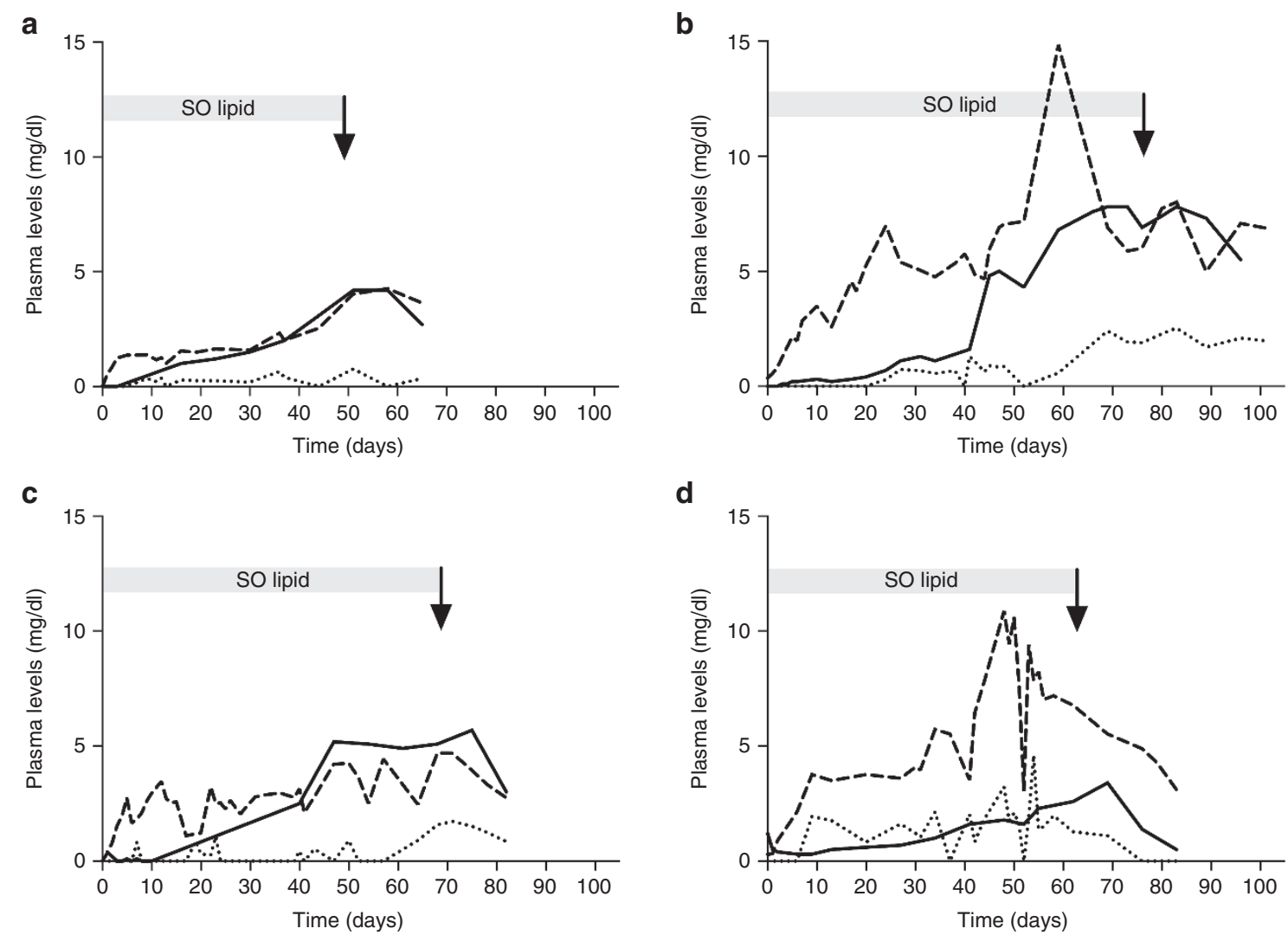

Figure 3. Serial phytosterol levels and conjugated bilirubin levels over time in four patients (a-d) who developed cholestasis (conjugated bilirubin $>2 \mathrm{mg} / \mathrm{dl}$ ). Initiation of soybean oil (SO) lipid is at time $=0$. The arrow indicates time of discontinuation of SO lipid. Changes in sitosterol (--), stigmasterol (...), and conjugated bilirubin (-) shown. 
diet had higher absorption of dietary phytosterols than children (20) and the absorption seen in infants and children were both higher than those previously reported in adults (21).

Although very preterm infants had increased sitosterol exposure during the first $5 \mathrm{~d}$ of SO lipid, maximum phytosterol levels and incidence of cholestasis were similar between groups. Because of our goal to determine phytosterol kinetic parameters after initiation of SO lipid, our study was not designed to assess factors that influence phytosterol concentrations throughout the course of SO lipid. However, a few things may have affected the metabolism of phytosterols during PN. First, enteral feeds were initiated earlier in the very preterm group. By increasing hormones such as secretin, glucagon, gastrin, and motilin, enteral feeds stimulate bile flow and gastrointestinal motility $(22,23)$. Even small feeds have been shown to promote intestinal epithelial cell growth, enzyme activity, and motility (24). Given these effects on bile flow and intestinal function, earlier enteral feeds may have played a role in increasing phytosterol elimination in the very preterm group. Second, it is important to note that infants in both gestational age categories received multiple medications. While the diuretic and surfactant therapy more frequently received by very preterm group have limited hepatic metabolism, many of the other medications are primarily metabolized by the liver and their ability to induce, inhibit, or modify hepatic ABCG5/ G8 transporters is unknown.

The implications for accumulated phytosterols are not fully understood, but support for toxicity comes from patients with sitosterolemia where loss of ABCG5/G8 function lead to toxic accumulation of plant sterols in the body that can lead to premature cardiovascular disease, hematological disorders, endocrine disruption, and possible liver cirrhosis $(25,26)$. Additionally, ABCG5/G8 knockout mice fed a high-phytosterol diet accumulate phytosterols in tissues and exhibit hepatic, cardiac, and hematologic dysfunction as well as growth failure and premature death $(27,28)$. While the contributions of phytosterol toxicity to PNALD are still being identified, other potential sequelae of phytosterol accumulation in infants receiving SO lipid are unknown and deserve further study.

This study has limitations. Similar to many pharmacokinetic studies in infants, accuracy of our kinetic models is limited by lack of repeated blood sampling due to the burden of phlebotomy in a vulnerable population. In order to maximize the number of time points for detailed kinetic analyses, we took advantage of the well-documented stability of phytosterols to a variety of conditions (29) and utilized all leftover blood samples that remained after completion of clinical laboratories. The different sampling times may have affected our kinetic analyses; however, we found no difference in our results after adjusting for random sampling in our analyses. Next, because of the study's objective to document the accumulation of phytosterols after initiation of SO lipid, we were unable to assess factors that disrupt steady-state. Lastly, our study was not designed to power the analysis of phytosterols in infants with cholestasis. This limits our ability to assess the correlation of phytosterol with markers of liver injury. A prospective study is currently underway to determine the kinetics of phytosterols throughout the duration of SO lipid therapy to evaluate these factors.

Modifying intravenous lipid therapy with lipid restriction of SO lipids or alternative lipid emulsions with different oil compositions have been proposed for the treatment and prevention of PNALD. In patients with established PNALD, restriction of SO lipid to $\leq 1 \mathrm{~g} / \mathrm{kg}$ per day has been associated with decreased bilirubin levels $(30,31)$. Similarly, changing from standard SO lipid therapy to a fish oil-based lipid emulsion such as Omegavan (Fresenius Kabi AG, Bad Homberg, Germany) has been shown to improve cholestasis and ameliorate the disease $(5,32,33)$. However, reports of SO lipid restriction in the prevention of PNALD have been conflicting (34-36), and the ability of Omegavan and other alternative lipid emulsions to prevent PNALD in infants remains unclear. Important issues limit use of these lipid modifications in infants. First, infants require fat for neural development during a critical period of rapid brain growth (12). Lipid restriction may have significant impact on the developing brain. There are no data regarding the long-term implications of lipid restriction in infants, and the impact of lipid restriction on neurodevelopment is unknown. Second, alternative lipid emulsions have not been approved for use in the United States. Moreover, the majority of alternative lipid emulsions are composed of some degree of soybean oil. For these reasons, infants receiving PN will likely continue to be exposed to phytosterols. Characterizing phytosterol metabolism and factors that predispose to excessive accumulation will help identify vulnerable populations and patient selection for different lipid emulsions. Given the potential toxicity of accumulated phytosterols, understanding phytosterol metabolism will continue to be of great importance in improving the safety of intravenous nutrition.

In conclusion, we believe this is the first description of the kinetics of phytosterols in infants receiving a soybean oil-based lipid emulsion. Our results demonstrate that phytosterols, particularly sitosterol and campesterol, rapidly accumulate in neonates during $\mathrm{SO}$ lipid infusions. With a short duration of SO lipid, very preterm infants have higher exposure to phytosterols, suggesting that they may have poorly developed mechanisms of metabolizing phytosterols. Thus, better understanding of the ontogeny of phytosterol metabolism may provide a mechanistic insight into the vulnerability of preterm infants to PNALD.

\section{METHODS}

The study was approved by the Institutional Review Board of Children's Hospital of Wisconsin (No. CHW 12/130) and conducted with written informed consent obtained from the parents or legal guardians. Between September 2012 and February 2013, infants admitted to the Children's Hospital of Wisconsin Neonatal Intensive Care Unit who received $\mathrm{PN} / \mathrm{SO}$ were screened for participation in this prospective pilot study. Inclusion criteria were anticipated need for SO lipid of at least $5 \mathrm{~d}$. Infants admitted to the Neonatal Intensive Care Unit who did not receive PN/SO lipid were enrolled as controls. Blood samples remaining after completion of clinical laboratories were collected on enrolled SO lipid-exposed and control patients for measurements of cholesterol and phytosterols (sitosterol, campesterol, and stigmasterol). Samples of SO lipid given to study subjects were also obtained for sterol analysis. Infants with known primary liver disease, anatomic 
liver abnormalities, metabolic disease, or known genetic or chromosomal defects were excluded from the study.

The primary outcome was the temporal change (kinetics) of plasma phytosterol concentrations. Accumulation was assessed using phytosterol levels obtained after SO lipid initiation until reaching a steadystate plasma concentration during SO lipid exposure. The average phytosterol level for a given day was used for infants who had more than one sterol level per day.

Decisions regarding medical care and nutrition provision were left to the discretion of the treating physicians. The initiation and advancement of parenteral and enteral nutrition for all study subjects followed pre-established Neonatal Intensive Care Unit guidelines. In general, intravenous lipid was initiated at a dosage of $2 \mathrm{~g} / \mathrm{kg}$ of body weight (bw) per day and advanced to a maximum of $3 \mathrm{~g} / \mathrm{kg}$ bw per day, amino acids initiated at $3 \mathrm{~g} / \mathrm{kg}$ bw per day and advanced to a maximum of $3.5-4 \mathrm{~g} / \mathrm{kg}$ bw per day in preterm infants and a maximum of $3 \mathrm{~g} / \mathrm{kg}$ bw per day in term infants, and carbohydrates initiated at a glucose infusion rate of $4-6 \mathrm{mg} / \mathrm{kg}$ per minute and advanced to a maximum of $10-12 \mathrm{mg} / \mathrm{kg}$ bw per minute. All neonates requiring PN received TrophAmine (B. Braun, Irvine, CA). Intravenous lipid emulsion was a SO lipid emulsion (Intralipid 20\%, Fresenius Kabi, Uppsala, Sweden). There were no episodes of sustained hyperglycemia or hypertriglyceridemia that required decreasing SO lipid infusion during the first $5 \mathrm{~d}$ of SO lipid therapy. Standard practice was to maintain SO lipid at $3 \mathrm{~g} / \mathrm{kg}$ bw per day until it was discontinued. Enteral nutrition was typically initiated at $10-20 \mathrm{ml} / \mathrm{kg}$ bw per day and advanced by $20-40 \mathrm{ml} / \mathrm{kg}$ bw per day as tolerated. If available, breast milk was the primary source of enteral nutrition, otherwise preterm or term formula was provided based on gestational age.

Demographic, clinical, and nutritional data were collected from the medical records. Demographic information included sex, race, gestational age at birth, weight, length, and head circumference at birth. Clinical data consisted of diagnoses, surgical procedures, culture-positive sepsis, and weight, length, and head circumference at discharge. Small for gestational age was defined as birth weight less than the tenth percentile for gestational age at birth. Early-onset and late-onset sepsis were defined as culture-positive sepsis occurring at $\leq 3 \mathrm{~d}$ and $>3 \mathrm{~d}$, respectively, in accordance with the American Academy of Pediatrics (37). Infants reached full feeds when they were able to tolerate $120 \mathrm{ml} / \mathrm{kg}$ bw per day of enteral feeds. Medications and laboratories including total and conjugated bilirubin, aspartate aminotransferase, alanine aminotransferase, alkaline phosphatase, and triglycerides were recorded. Cholestasis was defined as a serum conjugated bilirubin $>2 \mathrm{mg} / \mathrm{dl}$.

Nutritional data were collected daily for PN and enteral nutrition. Age at initiation of parenteral nutrition, initiation of enteral nutrition, and attaining full enteral feeds were recorded and duration of PN was calculated. Study data were collected and managed using the Research Electronic Data Capture (REDCap) system, a secure, webbased application used for data capture and management in clinical and translational research (38).

\section{Sterol Analyses}

All leftover blood samples remaining after completion of clinical laboratory testing were collected and analyzed for sterol concentrations. Plasma and SO lipid sterol analysis of cholesterol and phytosterols (sitosterol, campesterol, and stigmasterol) were evaluated by gas chromatography-mass spectrometry (GC/MS) as previously described $(39,40)$. In brief, $5 \alpha$-cholestane was added as an internal standard to plasma $(50-100 \mu \mathrm{l})$, saponified in ethanolic sodium hydroxide for $1 \mathrm{~h}$. Then, $1.5 \mathrm{ml}$ water was added and sterols extracted with three sequential portions of $9 \mathrm{ml}$ each of hexanes, pooled and dried. Dried sterol samples were derivatized as trimethylsilylethers, redissolved in $100 \mu \mathrm{l}$ ethyl acetate and $0.5 \mu \mathrm{l}$ samples injected splitless into a ThermoFinnegan GC/MS machine, using a 30m Restek column. Quantitation was based upon a standard curve from external standards, comprising of cholesterol, stigmasterol, sitosterol, and campesterol at varying concentrations $(39,40)$, using ultra-pure sterols obtained from Steraloids (Newport, RI). Parenteral cholesterol and phytosterol intake were calculated based on lipid emulsions analyzed for sterols similar to plasma sterols as described above. The lowest level of detection for sterol analysis using this technique is $0.01 \mathrm{mg} / \mathrm{dl}$.

\section{Pharmacokinetic Models}

Graphs of plasma phytosterol concentrations as a function of time after SO lipid initiation were used to evaluate the kinetics of phytosterol accumulation. For infants who had more than one episode of $\mathrm{PN} / \mathrm{SO}$ lipid, only sterol results obtained prior to restarting SO lipid were included in the analysis of sterol kinetics.

Phytosterol accumulation was described using nonlinear mixed effects models. We found that serial phytosterol levels after the initiation of SO lipid can be described well with a sigmoidal curve using the following equation:

$$
\text { Phytosterol }=C_{\mathrm{ss}} /\left(1+\exp \left(\left(T_{1 / 2}-\text { time }\right) / \mathrm{T}_{1 / 2-3 / 4}\right)\right)
$$

where $C_{s s}$ is steady-state concentration, $T_{t / 2}$ is the time to reach half of steady-state concentration, and $T_{1 / 2-3 / 4}$ is the time between $1 / 2$ and $3 / 4$ of steady-state concentration. Model simulation was carried out using the Levenberg-Marquardt algorithm (Matlab, Natick, MA). First, each subject was modeled individually to obtain individual kinetic parameters. To further examine the fit of the model, filtering was applied with a threshold for goodness-of-fit $R$ square of 0.1 and radius of the confidence interval for $C_{s s}$ of less than 20 .

Phytosterol exposure was evaluated using the area under the plasma concentration vs. time curve (AUC) between phytosterol sample points and computed using the trapezoidal rule. Because steadystate was achieved by $5 \mathrm{~d}$, the average AUC over the first $5 \mathrm{~d}$ of SO lipid exposure $\left(\mathrm{AUC}_{5}\right)$ was computed for each patient using the sum of the AUCs and dividing by the difference in days from the first sterol sample to the last sampled during the first $5 \mathrm{~d}$ of SO lipid.

\section{Statistical Analysis}

To evaluate the influence of immaturity on phytosterol kinetics, we hypothesized that the greatest difference in kinetic parameters would be seen in the most premature infants compared to more mature infants. Therefore, infants were classified and compared based on a gestational age category of very preterm (born $<28 \mathrm{wk}$ gestation) relative to those more mature (born $\geq 28$ wk gestation). To adjust for covariates, linear and stepwise linear regression (variables selected to stay with $P<0.05)$ were used to model the effect of gestational age group category on phytosterol $\mathrm{AUC}_{5}$, including race, sex, cumulative sitosterol intake for the first $5 \mathrm{~d}$ of SO lipid, and number of days receiving enteral feeds during the first $5 \mathrm{~d}$ of $\mathrm{SO}$ lipid as covariates. One patient did not have enough sterol samples for $\mathrm{AUC}_{5}$ computation and was eliminated from $\mathrm{AUC}_{5}$ analysis.

Stata Version 12.0 (Stata Corporation, College Station, TX) and SAS Version 9.2 (SAS Institute, Cary, NC) were used for statistical analysis. Unless otherwise stated, the data are expressed as median (IQR). Categorical data were compared using Fisher's exact test. Because the data were not normally distributed, continuous measures were compared using a nonparametric Wilcoxon-Mann-Whitney test. All tests were conducted as two-tailed tests with a $P$ value $<0.05$ considered statistically significant.

\section{SUPPLEMENTARY MATERIAL}

Supplementary material is linked to the online version of the paper at http://www.nature.com/pr

\section{ACKNOWLEDGMENTS}

The authors would like to thank Paula E. North and Andrew Webb for their assistance in facilitating sample collection. We also thank Joseph Emmer for his technical assistance with sterol analysis.

\section{STATEMENT OF FINANCIAL SUPPORT}

A portion of this work was supported by The Gerber Foundation (Fremont, MI) to T.H.N.-R., the National Center for Research Resources and the National Center for Advancing Translational Sciences, National Institutes of Health (Bethesda, MD) through grant number 8UL1TR000055 to T.H.N.-R., the Clinical and Translational Science Institute of Southeast Wisconsin (Bethesda, MD) through grant number UL1RR031973 to P.M.S., the Wisconsin 
Corporation for Biomedical Research (Milwaukee, WI) to S.B.P., and the Biomedical Laboratory Research and Development Program, Department of Veterans Affairs, Veterans Health Administration, Office of Research and Development (Washington, DC) to S.B.P. The contents of this manuscript are solely the responsibility of the authors and do not necessarily represent the official views of the various funding agencies.

Disclosure: The authors declare that they have no conflicts of interest to disclose.

\section{REFERENCES}

1. Hay WW Jr, Lucas A, Heird WC, et al. Workshop summary: nutrition of the extremely low birth weight infant. Pediatrics 1999;104:1360-8.

2. Ehrenkranz RA, Das A, Wrage LA, et al.; Eunice Kennedy Shriver National Institute of Child Health and Human Development Neonatal Research Network. Early nutrition mediates the influence of severity of illness on extremely LBW infants. Pediatr Res 2011;69:522-9.

3. Teitelbaum DH, Tracy T. Parenteral nutrition-associated cholestasis. Semin Pediatr Surg 2001;10:72-80.

4. Beale EF, Nelson RM, Bucciarelli RL, Donnelly WH, Eitzman DV. Intrahepatic cholestasis associated with parenteral nutrition in premature infants. Pediatrics 1979;64:342-7.

5. Premkumar $\mathrm{MH}$, Carter BA, Hawthorne KM, King K, Abrams SA. High rates of resolution of cholestasis in parenteral nutrition-associated liver disease with fish oil-based lipid emulsion monotherapy. J Pediatr 2013;162:793-798.e1.

6. Clayton PT, Bowron A, Mills KA, Massoud A, Casteels M, Milla PJ. Phytosterolemia in children with parenteral nutrition-associated cholestatic liver disease. Gastroenterology 1993;105:1806-13.

7. Kurvinen A, Nissinen MJ, Andersson S, et al. Parenteral plant sterols and intestinal failure-associated liver disease in neonates. J Pediatr Gastroenterol Nutr 2012;54:803-11.

8. Clayton PT, Whitfield P, Iyer K. The role of phytosterols in the pathogenesis of liver complications of pediatric parenteral nutrition. Nutrition 1998;14:158-64.

9. Whitfield PD, Clayton PT, Muller DP. Effect of intravenous lipid emulsions on hepatic cholesterol metabolism. J Pediatr Gastroenterol Nutr 2000;30:538-46.

10. Carter BA, Taylor OA, Prendergast DR, et al. Stigmasterol, a soy lipidderived phytosterol, is an antagonist of the bile acid nuclear receptor FXR. Pediatr Res 2007;62:301-6.

11. El Kasmi KC, Anderson AL, Devereaux MW, et al. Phytosterols promote liver injury and Kupffer cell activation in parenteral nutrition-associated liver disease. Sci Transl Med 2013;5:206ra137.

12. Cowan E, Nandivada P, Puder M. Fish oil-based lipid emulsion in the treatment of parenteral nutrition-associated liver disease. Curr Opin Pediatr 2013;25:193-200.

13. Ostlund RE Jr, McGill JB, Zeng CM, et al. Gastrointestinal absorption and plasma kinetics of soy Delta(5)-phytosterols and phytostanols in humans. Am J Physiol Endocrinol Metab 2002;282:E911-6.

14. Normén AL, Brants HA, Voorrips LE, Andersson HA, van den Brandt PA, Goldbohm RA. Plant sterol intakes and colorectal cancer risk in the Netherlands Cohort Study on Diet and Cancer. Am J Clin Nutr 2001;74: 141-8.

15. Valsta LM, Lemström A, Ovaskainen ML, et al. Estimation of plant sterol and cholesterol intake in Finland: quality of new values and their effect on intake. Br J Nutr 2004;92:671-8.

16. Iyer KR, Spitz L, Clayton P. BAPS prize lecture: New insight into mechanisms of parenteral nutrition-associated cholestasis: role of plant sterols. British Association of Paediatric Surgeons. J Pediatr Surg 1998;33:1-6.

17. Patel SB, Salen G, Hidaka $\mathrm{H}$, et al. Mapping a gene involved in regulating dietary cholesterol absorption. The sitosterolemia locus is found at chromosome 2p21. J Clin Invest 1998;102:1041-4.

18. Nghiem-Rao TH, Patel SB. Investigating sitosterolemia to understand lipid physiology. Clin Lipidol 2013;8:649-58.
19. Savini S, D’Ascenzo R, Biagetti C, et al. The effect of 5 intravenous lipid emulsions on plasma phytosterols in preterm infants receiving parenteral nutrition: a randomized clinical trial. Am J Clin Nutr 2013;98:312-8.

20. Mellies M, Glueck CJ, Sweeney C, Fallat RW, Tsang RC, Ishikawa TT. Plasma and dietary phytosterols in children. Pediatrics 1976;57:60-7.

21. Salen G, Ahrens EH Jr, Grundy SM. Metabolism of beta-sitosterol in man. J Clin Invest 1970;49:952-67.

22. Balistreri WF, Heubi JE, Suchy FJ. Immaturity of the enterohepatic circulation in early life: factors predisposing to "physiologic" maldigestion and cholestasis. J Pediatr Gastroenterol Nutr 1983;2:346-54.

23. Aynsley-Green A. Hormones and postnatal adaptation to enteral nutrition. J Pediatr Gastroenterol Nutr 1983;2:418-27.

24. Perdikis DA, Basson MD. Basal nutrition promotes human intestinal epithelial (Caco-2) proliferation, brush border enzyme activity, and motility. Crit Care Med 1997;25:159-65.

25. Lu K, Lee MH, Hazard S, et al. Two genes that map to the STSL locus cause sitosterolemia: genomic structure and spectrum of mutations involving sterolin-1 and sterolin-2, encoded by ABCG5 and ABCG8, respectively. Am J Hum Genet 2001;69:278-90.

26. Patel S, Salen G. Sitosterolemia: xenophobia for the body. In: Vissers MN, Kastelein JJP, Stroes ES, eds. Evidence-Based Management of Lipid Disorders. Harley, England: TFM Publishing Ltd, 2010:217-30.

27. Solca C, Tint GS, Patel SB. Dietary xenosterols lead to infertility and loss of abdominal adipose tissue in sterolin-deficient mice. J Lipid Res 2013;54:397-409.

28. McDaniel AL, Alger HM, Sawyer JK, et al. Phytosterol feeding causes toxicity in ABCG5/G8 knockout mice. Am J Pathol 2013;182:1131-8.

29. Engel LL, Brooks P. Cholesterol is stable. Steroids 1971;17:531-9.

30. Cober MP, Killu G, Brattain A, Welch KB, Kunisaki SM, Teitelbaum DH. Intravenous fat emulsions reduction for patients with parenteral nutritionassociated liver disease. J Pediatr 2012;160:421-7.

31. Cavicchi M, Beau P, Crenn P, Degott C, Messing B. Prevalence of liver disease and contributing factors in patients receiving home parenteral nutrition for permanent intestinal failure. Ann Intern Med 2000;132:525-32.

32. Gura KM, Duggan CP, Collier SB, et al. Reversal of parenteral nutrition-associated liver disease in two infants with short bowel syndrome using parenteral fish oil: implications for future management. Pediatrics 2006;118:e197-201.

33. Puder M, Valim C, Meisel JA, et al. Parenteral fish oil improves outcomes in patients with parenteral nutrition-associated liver injury. Ann Surg 2009;250:395-402.

34. Nehra D, Fallon EM, Potemkin AK, et al. A comparison of 2 intravenous lipid emulsions: interim analysis of a randomized controlled trial. JPEN 2013;37:498-505.

35. Rollins MD, Ward RM, Jackson WD, et al. Effect of decreased parenteral soybean lipid emulsion on hepatic function in infants at risk for parenteral nutrition-associated liver disease: a pilot study. J Pediatr Surg 2013;48:1348-56.

36. Sanchez SE, Braun LP, Mercer LD, Sherrill M, Stevens J, Javid PJ. The effect of lipid restriction on the prevention of parenteral nutrition-associated cholestasis in surgical infants. J Pediatr Surg 2013;48:573-8.

37. Polin RA; Committee on Fetus and Newborn. Management of neonates with suspected or proven early-onset bacterial sepsis. Pediatrics 2012;129:1006-15.

38. Harris PA, Taylor R, Thielke R, Payne J, Gonzalez N, Conde JG. Research electronic data capture (REDCap)-a metadata-driven methodology and workflow process for providing translational research informatics support. J Biomed Inform 2009;42:377-81.

39. Klett EL, Lu K, Kosters A, et al. A mouse model of sitosterolemia: absence of $\mathrm{Abcg} 8 /$ sterolin-2 results in failure to secrete biliary cholesterol. BMC Med 2004;2:5.

40. Chen J, Batta A, Zheng S, et al. The missense mutation in Abcg5 gene in spontaneously hypertensive rats (SHR) segregates with phytosterolemia but not hypertension. BMC Genet 2005;6:40. 